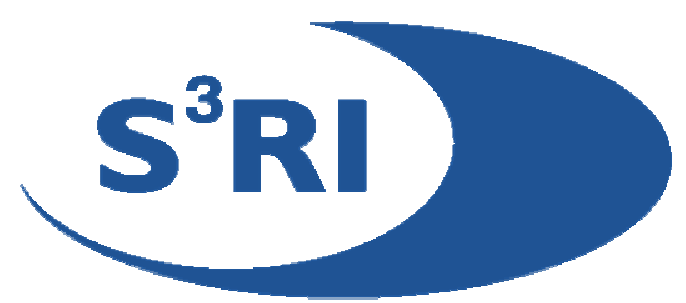

\title{
M-QuANTILE Models for SMall Area Estimation
}

\section{RAY CHAMBERS, NiKos TZAVIDIS}

\begin{abstract}
Small area estimation techniques are employed when sample data are insufficient for acceptably precise direct estimation in domains of interest. These techniques typically rely on regression models that use both covariates and random effects to explain variation between domains. However, such models also depend on strong distributional assumptions, require a formal specification of the random part of the model and do not easily allow for outlier robust inference. We describe a new approach to small area estimation that is based on modelling quantile-like parameters of the conditional distribution of the target variable given the covariates. This avoids the problems associated with specification of random effects, allowing inter-domain differences to be characterized by the variation of area-specific M-quantile coefficients. The proposed approach is easily made robust against outlying data values and can be adapted for estimation of a wide range of area specific parameters, including that of the quantiles of the distribution of the target variable in the different small areas. Results from two simulation studies comparing the performance of the M-quantile modelling approach with more traditional mixed model approaches are also provided.
\end{abstract}

\section{Southampton Statistical Sciences Research Institute Methodology Working Paper M05/07}




\title{
M-Quantile Models for Small Area Estimation
}

\author{
Ray Chambers \\ Southampton Statistical Sciences Research Institute, University of Southampton, Highfield, \\ Southampton, SO17 1BJ, UK \\ rc6@soton.ac.uk
}

Nikos Tzavidis

Southampton Statistical Sciences Research Institute, University of Southampton, Highfield, Southampton, SO17 1BJ, UK

ntzav@socsci.soton.ac.uk

\begin{abstract}
SUMMARY
Small area estimation techniques are employed when sample data are insufficient for acceptably precise direct estimation in domains of interest. These techniques typically rely on regression models that use both covariates and random effects to explain variation between domains. However, such models also depend on strong distributional assumptions, require a formal specification of the random part of the model and do not easily allow for outlier robust inference. We describe a new approach to small area estimation that is based on modelling quantile-like parameters of the conditional distribution of the target variable given the covariates. This avoids the problems associated with specification of random effects, allowing inter-domain differences to be characterized by the variation of area-specific M-quantile coefficients. The proposed approach is easily made robust against outlying data values and can be adapted for estimation of a wide range of area specific parameters, including that of the quantiles of the distribution of the target variable in the different small areas. Results from
\end{abstract}


two simulation studies comparing the performance of the M-quantile modelling approach with more traditional mixed model approaches are also provided.

Keywords: Influence functions; Multilevel models; Robust inference; Weighted least squares; Median estimation; Quantile regression.

\section{Introduction}

Sample surveys provide a cost effective way of obtaining estimates for characteristics of interest at both population and sub-population (domain) level. Such domains are typically defined in terms of geographic areas or by socio-demographic groups (Rao, 2003). An estimator of a domain characteristic is called direct if it is based only on data from sample units in the domain. A domain is large if the sample size within the domain is large enough to produce direct estimates of acceptable precision. In most practical applications, however, domain sample sizes are not large enough to allow direct estimation. The term "small areas" is typically used to describe such domains.

When direct estimation is not possible, one has to rely upon alternative methods for producing small area estimates. Such methods depend on the availability of population level auxiliary information related to the variable of interest and are commonly referred to as indirect or model-based methods. Model-based methods can be classified into two categories (a) methods based on fixed effects models, i.e. models that explain between area variation in the target variable using only the auxiliary information and (b) methods based on mixed (random) effects models that include area specific random effects to account for between area variation beyond that explained by the auxiliary information.

Mixed effects models are widely used in small area estimation (Fay and Herriot, 1979; Battese et. al., 1988). However, such models depend on parametric and distributional 
assumptions. They also require specification of the random part of the model, which is typically not straightforward. Furthermore, robust inference under these models is not straightforward.

In this paper we propose a new approach to small area estimation based on modelling quantile-like coefficients of the conditional distribution of the target variable given the covariates. Random effects are avoided. Instead, inter-domain variation is characterised by variation in area-specific values of these quantile-like coefficients. These new models are computationally straightforward to fit and have a number of practical advantages, including easy non-parametric specification and straightforward incorporation of survey weights. Outlier robust inference is also straightforward, as is estimation of other small area characteristics, e.g. medians and percentiles.

The structure of the paper is as follows: In Section 2 we summarise issues in small area estimation based on linear mixed effects models. In Section 3 we describe how quantile regression, and more generally $\mathrm{M}$-quantile regression, can be used to model a conditional distribution. In Section 4 we apply M-quantile regression to small area estimation. In Section 5 we discuss the differences between M-quantile and mixed effect models, highlighting the advantages of the M-quantile approach. In Section 6 we present results from two simulation studies comparing these alternative approaches when estimating small area means and medians. Finally, in Section 7 we summarise our main findings and give directions for further research.

\section{Linear Mixed Effects Models for Small Area Estimation}

In what follows we assume that a vector of $p$ auxiliary variables $\mathbf{x}_{i j}$ is known for each population unit $i$ in small area $j$ and that information for the variable of interest $y$ is available for units in the sample. The aim is to use these data is to estimate various area 
specific quantities, including (but not only) the small area mean $\bar{y}_{j}$ of $y$. However, the small area sample sizes are not large enough for reliable direct estimates, and one needs to use alternative methods of estimation that effectively "borrow strength" from all the small areas in order to produce an estimate for a particular small area. The most popular of these methods uses linear mixed effects models for this purpose. In the general case a linear mixed effects model has the following form

$$
y_{i j}=\mathbf{x}_{i j}^{T} \boldsymbol{\beta}+\mathbf{z}_{i j}^{T} \boldsymbol{\gamma}_{j}+\varepsilon_{i j}, i=1, \ldots, n, j=1, \ldots, d
$$

where $\boldsymbol{\gamma}_{j}$ denotes a vector of random effects and $\mathbf{z}_{i j}$ denotes a vector of auxiliary variables whose values are known for all units in the population. In addition, we usually assume that the random effects satisfy $\boldsymbol{\gamma}_{j} \sim N I D(\mathbf{0}, \Sigma), \varepsilon_{i j} \sim N I D\left(0, \omega^{2}\right)$ and $\boldsymbol{\gamma}_{j} \perp \varepsilon_{i j}$, where NID denotes "independently normally distributed". Estimation of the model parameters in (1) can be performed using Maximum Likelihood (ML) or Restricted Maximum Likelihood (REML) applied to the entire sample. Using the estimated fixed and random effects and the available auxiliary information we can then form domain specific estimates. For example, if we know the population size $N_{j}$ of small area $j$ then the mean $\bar{y}_{j}$ can be estimated by

$$
\hat{\bar{y}}_{j}=N_{j}^{-1}\left(\sum_{i \in s_{j}} y_{i j}+\sum_{i \in r_{j}} \mathbf{x}_{i j}^{T} \hat{\boldsymbol{\beta}}+\mathbf{z}_{i j}^{T} \hat{\boldsymbol{\gamma}}_{j}\right) .
$$

where a "hat" denotes an estimated quantity. This estimator is the more commonly known as Empirical Best Linear Unbiased Predictor (EBLUP) of $\bar{y}_{j}$ (Henderson, 1953; Kackar and Harville, 1981; Rao, 2003). Two special cases of this estimator may be noted. The first is where $\mathbf{z}_{i j}$ consists of a set of indicators for the small areas, in which case the $\boldsymbol{\gamma}_{j}$ are scalar small area effects. We refer to (2) in this case as the "random intercepts" estimator. The second is the more general situation where the $\mathbf{z}_{i j}$ include small area indicators as well as other covariates (which can be unit or area specific). We refer to (2) as the "random slopes" 
estimator in this case. In either case we note that the role of the random effects in the model is to characterise differences in the conditional distribution of $y$ given $x$ between the small areas. However, random effects modelling is not the only way we can develop such a characterisation. In the following section we describe quantile regression and related methods for modelling conditional distributions and in section 4 we use these methods to define an alternative approach to small area estimation.

\section{Modelling Quantiles}

The classical theory of linear statistical models is fundamentally a theory of conditional expectations. That is, a regression model summarises the behaviour of the mean of $y$ at each point in a set of $x$ 's (Mosteller and Tukey, 1977). Unfortunately, this summary provides a rather incomplete picture, in much the same way as the mean gives an incomplete picture of a distribution. It is usually much better to fit a family of regression models, each one summarising the behaviour of a different percentage point (quantile) of $y$ at each point in this set of $x$ 's. Such a modelling exercise is usually referred to as quantile regression.

\subsection{Quantile Regression}

The idea of modelling the quantiles of the conditional distribution of $y$ given $x$ has a long history in statistics. However the seminal paper by Koenker and Bassett (1978) is usually regarded as the first detailed development of this idea. In the linear case, quantile regression leads to a family (or "ensemble") of planes indexed by the value of the corresponding percentile coefficient $q \in(0,1)$. For each value of $q$, the corresponding model shows how $Q_{q}(x)$, the $q^{\text {th }}$ quantile of the conditional distribution of $y$ given $x$, varies with $x$. For example, when $q=0.5$ the quantile regression line shows how the median of this conditional distribution changes with $x$. Similarly, when $q=0.1$ this regression line separates the top $90 \%$ 
of the conditional distribution from the lower $10 \%$. A linear model for the $q^{\text {th }}$ conditional quantile of $y$ given a vector of covariates $\mathbf{x}$ is $Q_{q}(\mathbf{x})=\mathbf{x}^{T} \boldsymbol{\beta}_{q}$. The vector $\boldsymbol{\beta}_{q}$ is estimated by minimising $\sum_{i=1}^{n}\left|y_{i}-\mathbf{x}_{i}^{T} \mathbf{b}\right|\left\{(1-q) I\left(y_{i}-\mathbf{x}_{i}^{T} \mathbf{b} \leq 0\right)+q I\left(y_{i}-\mathbf{x}_{i}^{T} \mathbf{b}>0\right)\right\}$ with respect to $\mathbf{b}$. Solutions to this minimisation problem are usually obtained using linear programming methods (Koenker and D’Orey, 1987) and functions for fitting quantile regression now exist in standard statistical software, e.g. the. R statistical package (R Development Core Team, 2004).

Quantile regression is particularly useful when we have asymmetric data and the mean and median fits are different. A classic example of asymmetric data is Engel's (1857) analysis of the relationship between household food expenditure and household income. By plotting the different estimated quantile regression lines defined by Engel's data, Koenker and Hallock (2001) conclude that the dispersion of food expenditure increases with level of food expenditure as household income increases. Furthermore, the spacing of the estimated quantile regression lines show that the conditional distribution of food expenditure is skewed to the left in these data.

\subsection{M-Quantile Regression}

Quantile regression can be viewed as a generalisation of median regression. In the same way, expectile regression (Newey and Powell, 1987) is a "quantile-like" generalisation of mean (i.e. standard) regression. M-quantile regression (Breckling and Chambers, 1988) integrates these concepts within a common framework defined by a "quantile-like" generalisation of regression based on influence functions (M-regression). Since much of the development in this paper is based on the application of linear M-quantile regression, we now give a brief definition of this concept. 
The M-quantile of order $q$ for the conditional density of $y$ given $x$ is defined as the solution $Q_{q}(\mathbf{x} ; \psi)$ of the estimating equation $\int \psi_{q}(y-Q) f(y \mid \mathbf{x}) d y=0$, where $\psi$ denotes the influence function associated with the M-quantile. A linear M-quantile regression model is one where we assume that $Q_{q}(\mathbf{x} ; \psi)=\mathbf{x}^{T} \boldsymbol{\beta}_{\psi}(q)$. That is, we allow a different set of regression parameters for each value of $q$. For specified $q$ and $\psi$, estimates of these regression parameters can be obtained by solving the estimating equations

$$
\sum_{i=1}^{n} \psi_{q}\left(r_{i q \psi}\right) \mathbf{x}_{i}=\mathbf{0}
$$

where $r_{i q \psi}=y_{i}-\mathbf{x}_{i}^{T} \boldsymbol{\beta}_{\psi}(q), \psi_{q}\left(r_{i q \psi}\right)=2 \psi\left(s^{-1} r_{i q \psi}\right)\left\{q I\left(r_{i q \psi}>0\right)+(1-q) I\left(r_{i q \psi} \leq 0\right)\right\}$ and $s$ is a suitable robust estimate of scale, e.g. the MAD estimate $s=$ median $\left|r_{i q \psi}\right| / 0.6745$. We assume a Huber Proposal 2 influence function, $\psi(u)=u I(-c \leq u \leq c)+c \operatorname{sgn}(u)$. Provided $c$ is bounded away from zero, straightforward modification of widely available iteratively reweighted least squares software for fitting robust regression models (e.g. $r l m$ in $\mathrm{R}$, see Venables and Ripley, 2002) then leads to a solution of (3).

Why use M-quantile regression when "standard" quantile regression models can be fitted using standard statistical software? The answer is basically practical. Algorithms for fitting quantile regression models are based on linear programming methods and do not necessarily guarantee convergence and a unique solution. In contrast, the simple IRLS algorithm used to fit an M-quantile regression is guaranteed to converge to a unique solution (Kokic et. al., 1997) when a continuous monotone influence function (e.g. Huber Proposal 2 with $c>0$ ) is used. A further advantage of M-quantile regression is that it allows for more flexibility in modelling. For example, the tuning constant $c$ can be used to trade robustness for efficiency in the M-quantile regression fit, with increasing robustness/decreasing efficiency as $c \downarrow 0$ and 
we move towards quantile regression and decreasing robustness/increasing efficiency as $c \uparrow \infty$ and we move towards expectile regression.

A common problem for all "quantile-type" fitted regression planes is that they can cross over for different values of $q$. This lack of monotonicity is essentially a finite sample problem and is typically due to a combination of regression model misspecification, collinearity and highly influential data values. There is a large literature on "monotonizing" fitted quantile regression planes (e.g. Koneker, 1984; He, 1997). A simple method for scalar $x$ is described in Chambers (2005b), while Aragon et. al. (2005) describe a number of methods for monotonic nonparametric $\mathrm{M}$-quantile regression for general $\mathbf{x}$. In order to not detract from the interpretability of the M-quantile regression-based small area estimation approach described in the next section, we will not specifically address this issue in this paper.

\section{Using M-Quantile Regression to Measure Area Effects}

\subsection{Estimation of Small Area Characteristics}

Mixed effects models assume that variability associated with the conditional distribution of $y$ given $x$ can be at least partially explained by a pre-specified hierarchical structure, e.g. the small areas of interest. However, as we saw in the previous section, an alternative approach to modelling the variability in this conditional distribution is via M-quantile regression, which does not depend on a hierarchical structure. Consequently we index population units only by $i$ in what follows and, following Kokic et. al. (1997) and Aragon et. al. (2005), characterise conditional variability across the population of interest by the M-quantile coefficients of the population units. For unit $i$ with values $y_{i}$ and $\mathbf{x}_{i}$, this coefficient is the value $q_{i}$ such that $Q_{q_{i}}\left(\mathbf{x}_{i} ; \psi\right)=y_{i}$. Note that these M-quantile coefficients are determined at population level. Consequently, if a hierarchical structure does explain part of the variability in the population 
data, then we expect units within clusters defined by this hierarchy to have similar M-quantile coefficients. By definition,

$$
\begin{aligned}
\bar{y}_{j} & =N_{j}^{-1}\left(\sum_{i \in s_{j}} y_{i}+\sum_{i \in r_{j}} \mathbf{x}_{i}^{T} \boldsymbol{\beta}_{\psi}\left(q_{i}\right)\right) \\
& =N_{j}^{-1}\left(\sum_{i \in s_{j}} y_{i}+\sum_{i \in r_{j}} \mathbf{x}_{i}^{T} \boldsymbol{\beta}_{\psi}\left(\bar{q}_{j}\right)\right)+N_{j}^{-1} \sum_{i \in r_{j}} \mathbf{x}_{i}^{T}\left[\boldsymbol{\beta}_{\psi}\left(q_{i}\right)-\boldsymbol{\beta}_{\psi}\left(\bar{q}_{j}\right)\right]
\end{aligned}
$$

when the conditional M-quantiles follow a linear model. Here $\bar{q}_{j}=N_{j}^{-1} \sum_{i \in j} q_{i}$ is the average value of the M-quantile coefficients of the units in area $j$ and $s_{j}, r_{j}$ respectively denote the sampled and non-sampled units in area $j$. Typically the first term on the right hand side of the above expression will dominate, suggesting a predictor of $\bar{y}_{j}$ of the form

$$
\hat{\bar{y}}_{j}=N_{j}^{-1}\left(\sum_{i \in s_{j}} y_{i}+\sum_{i \in r_{j}} \mathbf{x}_{i}^{T} \hat{\boldsymbol{\beta}}_{\psi}\left(\hat{\bar{q}}_{j}\right)\right)
$$

where a "hat" represents an estimate of an unknown quantity. Note that alternative definitions of $\bar{q}_{j}$, and hence estimators of this quantity, are possible, e.g. the area $j$ median of the unit Mquantile coefficients. However, we do not explore them in this paper. We refer to $\bar{q}_{j}$ as the M-quantile coefficient of area $j$ in what follows.

Irrespective of how the M-quantile coefficient $\bar{q}_{j}$ for area $j$ is defined, (4) is equivalent to using $\mathbf{x}_{i}^{T} \boldsymbol{\beta}_{\psi}\left(\bar{q}_{j}\right)$ to predict the unobserved value $y_{i}$ for population unit $i \in r_{j}$. This suggests that predicted values for other small area characteristics can be also calculated using these unit level predictions. For example, the corresponding estimator of the finite population distribution function defined by $y$-values in area $j$ is

$$
\hat{F}_{j}(t)=N_{j}^{-1}\left(\sum_{i \in s_{j}} I\left(y_{i} \leq t\right)+\sum_{i \in r_{j}} I\left(\mathbf{x}_{i}^{T} \hat{\boldsymbol{\beta}}_{\psi}\left(\hat{\bar{q}}_{j}\right) \leq t\right)\right) .
$$


Estimates of the quantiles of the distribution of $y$ within area $j$ are easily derived from (5). Thus, the estimated median of the $y$-values in area $j$ is the median value of the set $\left\{y_{i} ; i \in s_{j}\right\} \cup\left\{\mathbf{x}_{i}^{T} \hat{\boldsymbol{\beta}}_{\psi}\left(\hat{\bar{q}}_{j}\right) ; i \in r_{j}\right\}$, with other estimated quantiles defined similarly.

In order to calculate any of (4) and (5) we need the estimated M-quantile coefficient for area $j$, i.e. $\hat{\bar{q}}_{j}$. Such an estimate will depend on the sample M-quantile coefficients, which we denote by $\left\{q_{i s} ; i \in s\right\}$, and which characterise the variation in the conditional distribution of $y$ given $\mathbf{x}$ in the sample in exactly the same way as the $q_{i}$ characterise this distribution in the population. In order to calculate the $q_{i s}$, we define a fine grid on the $(0,1)$ interval, and use the sample data to fit M-quantile regression lines at each value $q$ on this grid. The required $q_{\text {is }}$ values are then obtained by linear interpolation over this grid.

Provided the sampling method is non-informative given $\mathbf{x}, \hat{\bar{q}}_{j}$ can be calculated as the mean of the $q_{i s}$ values in area $j$. This is appropriate if $\bar{q}_{j}$ is defined as the mean value of the population $q_{i}$ values in area $j$. If a more robust definition of $\bar{q}_{j}$ is employed, say the median of these population values, then $\hat{\bar{q}}_{j}$ can be calculated as the median of the $q_{i s}$ values in area $j$. Alternatively, if a more complex method of sampling has been employed, and so the sample units are differentially weighted, then $\hat{\bar{q}}_{j}$ can be calculated as the corresponding weighted mean (or median) of the $q_{i s}$ values in area $j$. Note that a "pseudo-likelihood" approach, where sample inclusion probabilities are used to inversely weight the estimating equations (3), is not appropriate here because the sample "components" of the population level version of these equations actually depend on the entire population vector of $y$-values, and so asymptotically consistent estimation of the left hand side of the population version of (3) is impossible. 


\subsection{M-Quantile Sample Weighting}

For fixed $q$, the estimator of the M-quantile regression coefficient $\boldsymbol{\beta}_{\psi}(q)$ is $\hat{\boldsymbol{\beta}}_{\psi}(q)=\left(\mathbf{X}_{s}^{T} \mathbf{W}_{s}(q) \mathbf{X}_{s}\right)^{-1} \mathbf{X}_{s}^{T} \mathbf{W}_{s}(q) \mathbf{y}_{s}$, where $\mathbf{X}_{s}$ denotes the $n \times p$ matrix of sample covariate values and $\mathbf{y}_{s}$ is the $n$-vector of sample $y$-values. The diagonal matrix $\mathbf{W}_{s}(q)$ contains the final set of weights produced by the iteratively reweighted least squares algorithm (see section

3.2) used to compute $\hat{\boldsymbol{\beta}}_{\psi}(q)$. It immediately follows that (4) can be equivalently expressed as $\hat{\bar{y}}_{j}=N_{j}^{-1} \sum_{i \in s} w_{i j} y_{i}$, where

$$
\mathbf{w}_{j}=\left(w_{i j}\right)=\mathbf{1}_{s j}+\mathbf{W}_{s}\left(\hat{\bar{q}}_{j}\right) \mathbf{X}_{s}\left(\mathbf{X}_{s}^{T} \mathbf{W}_{s}\left(\hat{\bar{q}}_{j}\right) \mathbf{X}_{s}\right)^{-1} \mathbf{t}_{r j}
$$

Here $\mathbf{1}_{s j}$ is the $n$-vector with $i^{\text {th }}$ component equal to one whenever the corresponding sample unit is in area $j$ and is zero otherwise, and $\mathbf{t}_{r j}$ is the sum of the non-sample covariate values in area $j$.

Note that $\mathbf{X}_{s}^{\prime} \mathbf{w}_{j}=\mathbf{t}_{j}$, where $\mathbf{t}_{j}$ denotes the $p$-vector of population totals of the components of $\mathbf{x}$ in area $j$. That is, the weights defined by (6) are calibrated on the totals of the covariates in $\mathbf{x}$ within area $j$. An immediate consequence is that they sum to the population count $N_{j}$ in area $j$ whenever $\mathbf{x}$ contains an intercept term.

Provided that weights are purely a function of covariates, calibration on covariates is equivalent to unbiasedness under a population level linear model defined by these covariates (see Chambers, 2005a). The weights defined by (6) depend on the sample $y$-values and so this condition technically does not hold. If we treat these weights as fixed, then weighting via (6) leads to estimators that are approximately unbiased for $\bar{y}_{j}$ under the model $E\left(y_{i} \mid \mathbf{x}_{i}\right)=\mathbf{x}_{i}^{\prime} \boldsymbol{\beta}$. However, this does not mean that (6) (or equivalently (4)) leads to an estimator that is unbiased within area $j$ (i.e. is conditionally unbiased). For example, suppose $E\left(y_{i} \mid \mathbf{x}_{i}, i \in j\right)=E_{c}\left(y_{i} \mid \mathbf{x}_{i}\right)=\mathbf{x}_{i}^{\prime} \boldsymbol{\beta}_{j}$, then 


$$
E_{c}\left(N_{j}^{-1} \sum_{i \in s} w_{i j} y_{i}-\bar{y}_{j}\right) \approx N_{j}^{-1}\left(\sum_{k} \sum_{i \in s_{k}} w_{i j} \mathbf{x}_{i}^{\prime} \boldsymbol{\beta}_{k}-\sum_{i \in j} \mathbf{x}_{i}^{\prime} \boldsymbol{\beta}_{j}\right) \neq \mathbf{0}
$$

where the summation over $k$ on the right hand side above is over the set of areas represented in the sample.

\subsection{Estimation of Mean Squared Error}

We use the fact that the M-quantile estimator of $\boldsymbol{\beta}_{\psi}(q)$ is linear in the sample values of $y$ to develop a "plug-in" estimator of the mean squared error of (4). Estimation of the mean squared error of the distribution function estimator (5) is more complex and will be discussed elsewhere. Note that our "plug-in" approach assumes $\hat{\bar{q}}_{j}$ is constant, which leads to a first order approximation to the actual mean squared error of (4).

Mean squared error estimation of $\hat{\bar{y}}_{j}$ can be carried out using standard methods for robust estimation of the mean squared error of unbiased weighted linear estimators (Royall and Cumberland, 1978). That is, the prediction variance of (4) can be approximated by

$$
\operatorname{Var}\left(\hat{\bar{y}}_{j}-\bar{y}_{j}\right) \approx N_{j}^{-2}\left(\sum_{i \in s} u_{i j}^{2} \operatorname{Var}\left(y_{i}\right)+\sum_{i \in r_{j}} \operatorname{Var}\left(y_{i}\right)\right)
$$

where we treat the weights (6) as fixed. Here $\mathbf{u}_{j}=\left(u_{i j}\right)=\mathbf{W}_{s}\left(\hat{\bar{q}}_{j}\right) \mathbf{X}_{s}\left(\mathbf{X}_{s}^{T} \mathbf{W}_{s}\left(\hat{\bar{q}}_{j}\right)^{-1} \mathbf{X}_{s}\right)^{-1} \mathbf{t}_{r j}$. The basic problem then becomes one of interpreting $\operatorname{Var}\left(y_{i}\right)$ in this approximation. Following Chambers (2005a), we can be conservative and take this variance to be unconditional (i.e. not specific to the area from which $y_{i}$ is drawn) and hence replace $\operatorname{Var}\left(y_{i}\right)$ in the first (sample) term on the right hand side above by $\left(y_{i}-\mathbf{x}_{i}^{T} \hat{\boldsymbol{\beta}}_{\psi}(0.5)\right)^{2}$ and the second term by $\frac{N_{j}-n_{j}}{n_{j}-1} \sum_{i \in s_{j}}\left(y_{i}-\mathbf{x}_{i}^{T} \hat{\boldsymbol{\beta}}_{\psi}(0.5)\right)^{2}$. We refer to this approach as the population level residuals approach. Alternatively, we can interpret $\operatorname{Var}\left(y_{i}\right)$ conditionally (i.e. specific to the area $k$ from 
which $y_{i}$ is drawn) and hence replace $\operatorname{Var}\left(y_{i}\right)$ in the first (sample) term on the right hand side above by $\left(y_{i}-\mathbf{x}_{i}^{T} \hat{\boldsymbol{\beta}}_{\psi}\left(\hat{\bar{q}}_{k}\right)\right)^{2}$ and the second term by $\frac{N_{j}-n_{j}}{n_{j}-1} \sum_{i \in s_{j}}\left(y_{i}-\mathbf{x}_{i}^{T} \hat{\boldsymbol{\beta}}_{\psi}\left(\hat{\bar{q}}_{j}\right)\right)^{2}$. We refer to this approach as the area level residuals approach. Our estimator of the prediction variance of (4) is therefore either

$$
\hat{V}_{j}=\sum_{i \in s} \theta_{i j}\left(y_{i}-\mathbf{x}_{i}^{T} \hat{\boldsymbol{\beta}}_{\psi}(0.5)\right)^{2}
$$

or

$$
\hat{V}_{j}=\sum_{k} \sum_{i \in s_{k}} \theta_{i j}\left(y_{i}-\mathbf{x}_{i}^{T} \hat{\boldsymbol{\beta}}_{\psi}\left(\hat{\bar{q}}_{k}\right)\right)^{2}
$$

where $\theta_{i j}=N_{j}^{-2}\left(u_{i j}^{2}+I(i \in j)\left(N_{j}-n_{j}\right) /\left(n_{j}-1\right)\right)$. In order to estimate the mean squared error of this estimator we add an estimate of the squared conditional bias of (4) to (8). This bias estimate follows from (7) and is given by

$$
\hat{B}_{j}=N_{j}^{-1}\left(\sum_{k} \sum_{i \in s_{k}} w_{i j} \mathbf{x}_{i}^{\prime} \hat{\boldsymbol{\beta}}\left(\hat{\bar{q}}_{k}\right)-\sum_{i \in j} \mathbf{x}_{i}^{\prime} \hat{\boldsymbol{\beta}}\left(\hat{\bar{q}}_{j}\right)\right) .
$$

Our final estimator of the mean squared error of (4) is therefore obtained by adding the square of (9) to (8):

$$
\hat{M}_{j}=\hat{V}_{j}+\hat{B}_{j}^{2}
$$

\section{M-Quantile Models vs. Mixed Effects Models for Small Area Estimation}

What are the advantages (and disadvantages) of adopting an M-quantile modelling approach compared with a mixed effects modelling approach to small area estimation? A linear mixed effects model consists of a fixed part, defined by a linear model for $E(y \mid \mathbf{x})$, whose specification is the same for all small areas and a random part that is specific to a particular area. The random part of the model effectively accounts for residual between-area variation 
beyond that explained by $\mathbf{x}$ (i.e. it corrects for misspecification of $E(y \mid \mathbf{x})$ ). In contrast, the M-quantile modelling approach captures this residual between-area variation by the deviation of the area specific M-quantile regression coefficient $\hat{\boldsymbol{\beta}}_{\psi}\left(\hat{\bar{q}}_{j}\right)$ from the "median" M-quantile coefficient $\hat{\boldsymbol{\beta}}_{\psi}(0.5)$. This allows us to write the M-quantile regression model in a form that mimics the mixed effects form, via the identity

$$
y_{i j}=\mathbf{x}_{i}^{T} \boldsymbol{\beta}_{\psi}(0.5)+\mathbf{x}_{i}^{T}\left(\boldsymbol{\beta}_{\psi}\left(\bar{q}_{j}\right)-\boldsymbol{\beta}_{\psi}(0.5)\right)+\left(y_{i j}-\mathbf{x}_{i}^{T} \boldsymbol{\beta}_{\psi}\left(\bar{q}_{j}\right)\right) .
$$

The middle term on the right hand side of (11) can be interpreted as a pseudo-random effect for area $j$, while the third term represents the within area residual. From this point of view, Mquantile modelling will work provided this pseudo-random effect actually captures area effects.

It is tempting to use (11) to argue that M-quantile modelling for small areas is essentially a distribution free version of random slopes modelling. However, this is not true. The reality is that M-quantile model fit to an area can look "like" the corresponding random intercepts or the random slopes fit for the area depending on the correlations between the actual random effects in the data. To see this, we note that the individual M-quantile coefficients $q_{i}$ are independent of the corresponding $\mathbf{x}_{i}$ provided the model for $y$ given $\mathbf{x}$ is properly specified. They are also computed without reference to an area classification. Imposing such a classification may therefore induce a relationship between the sample $q_{i}$ values and their $\mathbf{x}_{i}$ values, where the form of this relationship will depend on the characteristics of the mixed model. Thus, if there is a medium to strong correlation (positive or negative) between the underlying random effects in the data (e.g. between the intercept and slope coefficients), then we would expect to see a corresponding relationship between the $q_{i}$ and $\mathbf{x}_{i}$ values in each area and hence an M-quantile fit within the areas that looks like the corresponding random slopes fit. Conversely, if this correlation is weak then the relationship between the $q_{i}$ and the 
$\mathbf{x}_{i}$ values in each area will also be weak and the M-quantile fit to the different areas will look like the corresponding random intercepts fit in these areas. That is, although one can interpret the presence of slope variation in the M-quantile fit to the different areas as indicative of random slopes in the data, one cannot interpret the lack of slope variation in the M-quantile fit as indicative of a random intercepts specification.

Using M-quantile models for small area estimation has a number of advantages over the use of mixed models. To start, M-quantile models are free of strong distributional assumptions. In contrast, mixed models typically impose Gaussian assumptions on the random effects. Although the use of normality assumptions is mathematically convenient, there is no reason to assume that random effects are normally distributed.

A second advantage is ease of model specification. An important step in mixed effects modelling is the specification of the random part of the model. This depends on the judicious use of diagnostic tests such as likelihood ratio tests. Although model selection for the random intercepts model is relatively straightforward, the same cannot be said when we are fitting a random slopes model, particularly when there are a large number of explanatory variables. Model specification for M-quantile models, on the other hand, only requires application of standard modelling methods to identify $\mathbf{x}$.

The third advantage is that M-quantile models allow for outlier robust inference using widely available M-estimation software. Although outlier robust estimation methods for mixed models have been developed (Richardson and Welsh, 1996), software implementing this methodology is not widely available.

Finally, we note that nonparametric specification of the relationship between $y$ and $\mathbf{x}$ is easily accommodated within the M-quantile modelling approach (see Aragon et. al., 2005). We are not aware of similar nonparametric approaches to fitting mixed models. 
However, M-quantile modelling has its drawbacks as well. Chief among these is that it requires data where the M-quantile $Q_{q}(\mathbf{x} ; \psi)$ for the conditional distribution of $y$ given $\mathbf{x}$ is well defined. This is true where $y$ is continuous or at least ordinal, but is more problematic where $y$ is nominal. Mixed models, on the other hand, are easily generalised (e.g. via generalised linear mixed models) to this case. Further work needs to be done on extending the M-quantile concept to allow modelling the conditional distribution of $y$ given $\mathbf{x}$ when $y$ is nominal. Another area where problems arise is where the response variable is multivariate. Since there is no unique way of ordering multivariate data, some agreed way of defining a direction for ordering in two or more dimensions is needed before one can define the Mquantiles of the conditional distribution of a multivariate $\mathbf{y}$ given $\mathbf{x}$. Breckling and Chambers (1988) describe such a multivariate generalisation, but its regression properties are unknown. Finally, it should be recognised that M-quantile modelling will never be as efficient as mixed modelling when the assumptions of the latter approach are in fact true. In our opinion, the primary role of M-quantile models is to provide a robust alternative to mixed models when one has reservations about the assumptions inherent in the latter.

\section{Simulation Studies}

In this section we present results from two simulation studies that were used to compare the M-quantile and mixed model approaches to small area estimation. The first was a modelbased simulation in which small area population and sample data were simulated based on a two level hierarchical linear model. The second was a design-based simulation in which a fixed population containing a number of small areas was repeatedly sampled, holding the sample size in each small area fixed. Four different methods of small area estimation were applied to the sample data obtained in the simulations, based on fitting linear models under (a) a random intercepts specification, (b) a random slopes specification, (c) a M-quantile 
approach with $c=1.345$ (referred to as "M-Quantile" below), and (d) a M-quantile approach with $c=2 \times 10^{5}$ (referred to as "Expectile" below). Both (a) and (b) were based on fitting a linear mixed model to the sample data using the default settings of the lme function (Bates and Pinheiro, 1998) in R. Similarly, the M-quantile regression fits underpinning (c) and (d) were obtained using a straightforward modification of the $r m$ function (Venables and Ripley, 2002) in R. Mean estimates were then calculated using (2) and (4), while median estimates under the mixed effects model (1) were calculated using

$$
\overline{\text { median }_{M M}\left(\mathbf{y}_{j}\right)}=\operatorname{median}\left[\left\{y_{i} ; i \in s_{j}\right\} \cup\left\{\mathbf{x}_{i}^{T} \hat{\boldsymbol{\beta}}+\mathbf{z}_{i}^{T} \hat{\boldsymbol{\gamma}}_{j} ; i \in r_{j}\right\}\right] \text {. }
$$

Median estimates under the M-quantile approach were calculated similarly, using

$$
\overline{\operatorname{median}_{M Q}\left(\mathbf{y}_{j}\right)}=\operatorname{median}\left[\left\{y_{i} ; i \in s_{j}\right\} \cup\left\{\mathbf{x}_{i}^{T} \hat{\boldsymbol{\beta}}_{\psi}\left(\hat{\bar{q}}_{j}\right) ; i \in r_{j}\right\}\right]
$$

\subsection{Model-Based Simulations}

In each simulation we generated $N=232,500$ population values of $x$ and $y$ in $H=30$ small areas with $N_{h}=500 h$ in area $h$. For each area $h$ we took a simple random sample (without replacement) of size $n_{h}=30$, leading to an overall sample size of $n=900$. The sample values of $y$ and the population values of $x$ were then used to estimate the mean and the median of $y$ for each area and the resulting estimation errors computed. This process was repeated 1000 times. The population mean $\mu_{h}$ of the $x$-values in small area $h$ was chosen at random and without replacement from the integers between 40 and 200, and was held fixed over all simulations. Individual $x$-values within area $h$ were drawn independently at each simulation as $N\left(\mu_{h}, \mu_{h}^{2} / 36\right)$, with $y$-values calculated similarly as $y_{i}=5+x_{i}+10 \gamma_{0 h}+\theta \gamma_{1 h} x_{i}+40 e_{i}$, where the $e_{i}$ were drawn independently from a $N(0,1)$ distribution. The small area effects $\gamma_{0 h}$ and $\gamma_{1 h}$ were independently drawn from a bivariate standard normal distribution with 
$\operatorname{cor}\left(\gamma_{0 h}, \gamma_{1 h}\right)=\rho$. Four scenarios were simulated, defined by $(\theta, \rho)=(0,0),(1,0),(1,0.8)$ and $(1,-0.8)$

Biases and mean squared errors over these simulations, averaged over the 30 areas, are set out in Table 1. These show that in this situation, where the random effects model (1) underpinning the random intercepts and random slopes approaches (a) and (b) respectively are in fact true, then these methods are, unsurprisingly, most efficient. However, the M-quantile and Expectile methods (c) and (d) are almost equally efficient in the case of estimation of the small area means, and, in the case of estimation of small area medians, the Expectile method (d) was again almost as efficient, with the M-quantile method performing equivalently in terms of bias, but less efficiently in terms of MSE. This can be explained by the normality of the simulated within area data in our experiments (ensuring similarity of means and medians) and the fact that under normality the robust M-quantile regression method (c) is less efficient than the non-robust Expectile method (d). In addition, these experiments show relative differences in performance of the four methods that seem almost invariant to the structure of the particular mixed model underpinning the population $Y$-values.

\subsection{Design-Based Simulations}

The data on which these simulations were based were the same as used in Chambers (2005a) and were obtained from a sample of 1652 broadacre farms spread across 29 regions (Region) of Australia, with the $y$-variable of interest equal to the Total Cash Costs (TCC) of the farm business in the reference year. Auxiliary information available for each farm included the total area of the farm in hectares (FarmArea) and the climatic zone in which the farm is situated. This information was used to classify the farms into six SizeZone strata $(1=$ pastoral zone and a farm area of 50000 hectares or less, 2 = pastoral zone and a farm area of more than 50000 hectares, 3 = wheat-sheep zone and a farm area of 1500 hectares or less, $4=$ 
wheat-sheep zone and a farm area of more than 1500 hectares, $5=$ high rainfall zone and a farm area of 750 hectares or less, $6=$ high rainfall zone and a farm area of more than 750 hectares). Individual (farm) level values for FarmArea, SizeZone and Region were assumed known at the population level.

The aim of this simulation study was to compare estimation of regional means and medians of TCC under repeated sampling using both mixed effects models and M-quantile models. The study itself was implemented as follows:

- $\quad$ The same population of 81982 farms described in Chambers (2005a) was used. This was obtained by "bootstrapping" the original sample of 1652 farms by sampling with replacement and with probability proportional to a farm's sample weight within each of the 29 regions. Scatterplots of the distribution of $y$ and $x$ in this population are set out in Chambers (2005a) and show that this population is highly heteroskedastic, with many outlying values.

- $\quad 500$ independently stratified random samples of the same size as the original sample were selected from this simulated population. These were the same samples as used in Chambers (2005a). Stratum (i.e. region) sample sizes were fixed to be the same as in the original sample. Table 2 shows the population and sample size, as well as the mean and median of TCC within each region.

The same second order fixed effects specification was used by all estimation methods, defined by the main effects and interactions for the Farmarea and SizeZone variables, with a random slopes specification implemented by assuming a random slope for Farmarea.

The results set out in Tables 3 and 4 focus on estimation of regional means. These show that estimation via mixed models typically leads to smaller relative biases (Table 3). However, these smaller biases come at a cost of significantly higher variability. Consequently when we compare relative root mean squared errors (Table 4) we see that all four methods are 
essentially the same. The larger bias of the M-quantile approach is due to the presence of outlying values in the dataset. Essentially, such outlying values receive small weights under the M-quantile approach and so bias is introduced when the target of inference is the small area mean. In contrast the smaller variation in weights under the less robust Expectile approach means that this bias is reduced.

A very different picture emerges when we turn to estimation of regional medians, however. Now we see that both M-quantile methods exhibit much smaller relative bias (Table 5) as well as relative median bias (Table 6) than the methods based on application of mixed models. Furthermore, these lower biases are accompanied by lower variances, leading to relative root mean squared errors (Table 7) for the M-quantile methods that are around a half of those observed for the mixed model-based methods.

Finally, in Table 8 we show the coverage performances of confidence intervals for the regional means based on M-quantile and Expectile estimates and the MSE estimator (10). Here we see that the larger design-biases of the M-quantile method (see Table 3) lead to poorer coverage than the Expectile method. As expected, using the population residual-based variance estimator (8a) in (10) leads to conservative estimates of MSE. However, this conservatism persists when we use the area residual-based variance estimator $(8 b)$ in $(10)$, which is somewhat surprising. Overall, provided the M-quantile method used is itself not very biased, it seems clear that the MSE estimator (10) is generally conservative, indicating that further research is required to identify a more accurate method of MSE estimation under Mquantile sample weighting.

\section{Concluding Remarks}

In this paper we introduce M-quantile models for small area estimation. These models offer a natural way of modeling between area variability in data without imposing prior 
assumptions about the source of this variability. In particular, with M-quantile models there is no need to explicitly specify the random components of the model, with inter-area differences captured via area-specific M-quantile coefficients instead. As a consequence, the M-quantile approach reduces the need for parametric assumptions and provides great flexibility in model selection. In addition, estimation and outlier robust inference under these models is straightforward. The proposed approach appears to be suitable for estimating a wide range of parameters and our simulation results show that it is a reasonable alternative to mixed effects models for small area estimation.

The M-quantile approach also opens new areas for research in the practical use of mixed models. For example, the existence of hierarchical effects of a particular type may be checked using diagnostic tests based on the M-quantile coefficients associated with this hierarchy. Alternatively, tests for the significance or otherwise of the coefficients of the M-quantile model may be useful in deciding on an appropriate formulation for the random part of a mixed effects model.

An important problem in small area estimation is the impact of changing small area geographies on the estimates. This is difficult to resolve using mixed effects models since the random effects in these models are geography dependent, and so a change in the geography requires new random effects to be estimated. Such problems do not arise with M-quantile models since M-quantile coefficients are associated with individual population units and so do not change when a new geography is introduced. This allows the corresponding M-quantile coefficients for the new geography to be obtained by simply re-aggregating these fixed unit level M-quantile coefficients.

As in any branch of statistics, it is important for the data analyst involved in small area estimation to decide on an appropriate target of inference. For example, with highly skewed distributions one might assume that the median is a more appropriate measure of central 
tendency than the mean. In such a case, a robust approach like the M-quantile one will outperform the mixed effects approach. If however the mean is chosen as the target of inference, then our simulation results indicate that although in general an estimator based on a mixed effects model will have lower bias, all approaches are close in mean squared error terms. Nevertheless, low bias is typically a desirable property, and further research is ongoing to modify the M-quantile approach in such situations to decrease its bias. This research will be reported elsewhere.

\section{Acknowledgements}

The research described in this paper was carried out under grant H333250030 of the Economic and Social Research Council.

\section{References}

Aragon, Y., et. al. (2005). Conditional ordering using nonparametric expectiles. Working Paper M05/01, Southampton Statistical Sciences Research Institute.

Bates, D.M. and Pinheiro, J.C. (1998). Computational methods for multilevel models. Available in PostScript or PDF formats at http://franz.stat.wisc.edu/pub/NLME/

Battese, G.E., et. al. (1988). An error component model for prediction of county crop areas using survey and satellite data. Journal of the American Statistical Association 83, 28-36.

Breckling, J. and Chambers, R. (1988). M-quantiles. Biometrika 75, 761-71.

Chambers, R.L. (2005a). Calibrated weighting for small area estimation. Working Paper M05/04, Southampton Statistical Sciences Research Institute.

Chambers, R.L. (2005b). What if .... Robust prediction intervals for unbalanced samples. Working Paper M05/05, Southampton Statistical Sciences Research Institute. 
Engel, E. (1857). Die Produktions- und Konsumverhältnisse des Königreichs Sachsen. Zeitschrift des Statistischen Büros des Königlich Sächsischen Ministeriums des Inneren, 8 , $1-54$.

Fay, R.E. and Herriot, R.A. (1979). Estimation of income from small places: An application of James-Stein procedures to census data. Journal of the American Statistical Association 74, 269-77.

He, X. (1997). Quantile curves without crossing. The American Statistician, 51, 186-92.

Henderson, C.R. (1953). Estimation of variance and covariance components, Biometrics, 9, 226-52.

Kackar, R.N. and Harville, D.A. (1981). Unbiasedness of two-stage estimation and prediction procedures for mixed linear models. Communications in Statistics, Series A, 10, 1249-61.

Koenker, R. and Bassett, G. (1978). Regression quantiles. Econometrica, 46, 33-50.

Koneker, R. (1984). A note on L-estimators for linear models. Statistics and Probability Letters, 2, 323-25

Koenker R. and D’Orey, V. (1987). Computing regression quantiles, Applied Statistics, 36, 383-93.

Koenker, R. and Hallock, K.F. (2001). Quantile regression: An introduction, Journal of Economic Perspectives, 51, 143-56.

Kokic, P. et. al. (1997). A measure of production performance, Journal of Business and Economic Statistics, 15, 445-51.

Mosteller, F. and Tukey, J.W. (1977). Data Analysis and Regression. A Secondary Course in Statistics, Reading, MA: Addison-Wesley.

Newey, W.K. and Powell, J.L. (1987). Asymmetric least squares estimation and testing, Econometrica, 55, 819-47. 
R Development Core Team (2004). R: A language and environment for statistical computing. R Foundation for Statistical Computing, Vienna, Austria. URL: http://www.R-project.org. Rao, J.N.K. (2003). Small Area Estimation. New York: Wiley.

Richardson, A.M. and Welsh, A.H. (1996). Covariate screening in mixed linear models, Journal of Multivariate Analysis, 58, 27-54.

Royall, R.M. and Cumberland, W.G. (1978). Variance estimation in finite population sampling. Journal of the American Statistical Association, 73, 351 - 58.

Venables, W.N. and Ripley, B.D. (2002). Modern Applied Statistics with S. New York: Springer. 
Table 1 Simulation results for two level linear populations, averaged over 30 small areas.

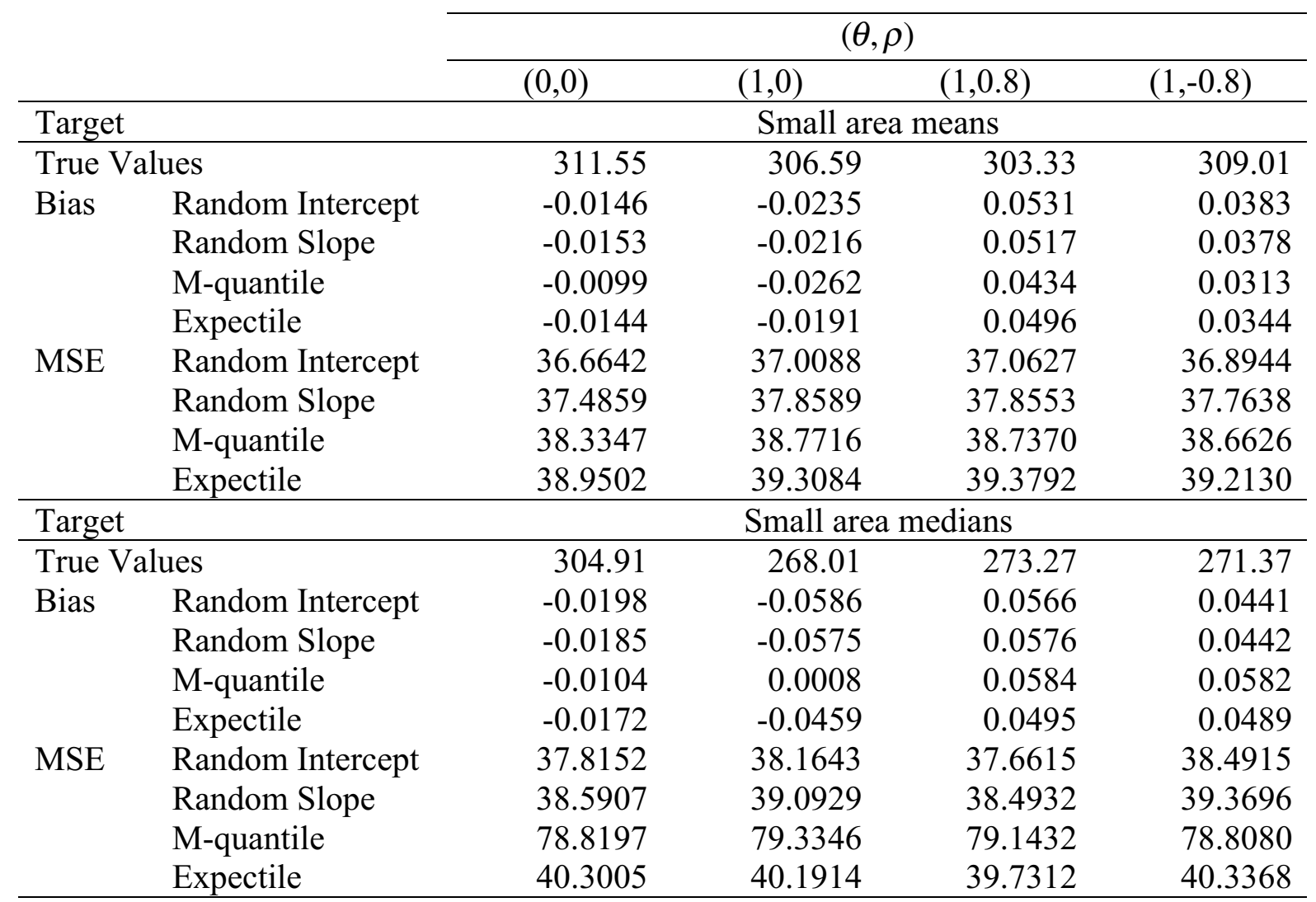


Table 2 Regional population and sample sizes and true values of mean and median of TCC in simulation study

\begin{tabular}{|c|c|c|c|c|}
\hline Region & Population & Sample & Mean & Median \\
\hline 1 & 79 & 6 & 467964 & 289858 \\
\hline 2 & 115 & 10 & 171414 & 64612 \\
\hline 3 & 189 & 30 & 670927 & 314577 \\
\hline 4 & 330 & 25 & 186984 & 165463 \\
\hline 5 & 388 & 36 & 208142 & 119817 \\
\hline 6 & 465 & 19 & 130316 & 41856 \\
\hline 7 & 604 & 36 & 302583 & 186163 \\
\hline 8 & 729 & 40 & 242836 & 216277 \\
\hline 9 & 737 & 30 & 179112 & 143205 \\
\hline 10 & 964 & 30 & 180467 & 177278 \\
\hline 11 & 1586 & 51 & 116965 & 93720 \\
\hline 12 & 1778 & 62 & 114442 & 67497 \\
\hline 13 & 1984 & 55 & 96162 & 74578 \\
\hline 14 & 2182 & 47 & 233171 & 213057 \\
\hline 15 & 2607 & 79 & 97839 & 62762 \\
\hline 16 & 2683 & 60 & 93202 & 42624 \\
\hline 17 & 2689 & 60 & 84790 & 61114 \\
\hline 18 & 2847 & 34 & 36979 & 26075 \\
\hline 19 & 3056 & 74 & 101101 & 65662 \\
\hline 20 & 3139 & 51 & 87919 & 45276 \\
\hline 21 & 3910 & 73 & 78509 & 34195 \\
\hline 22 & 4486 & 117 & 164889 & 92005 \\
\hline 23 & 4550 & 80 & 86218 & 62144 \\
\hline 24 & 4587 & 95 & 184153 & 109985 \\
\hline 25 & 5368 & 83 & 198156 & 133334 \\
\hline 26 & 5528 & 103 & 105152 & 72378 \\
\hline 27 & 6489 & 108 & 134169 & 88891 \\
\hline 28 & 6980 & 81 & 95617 & 66269 \\
\hline 29 & 10933 & 77 & 66285 & 45867 \\
\hline
\end{tabular}


Table 3 Relative biases of estimates of regional means of TCC in simulation study. Regions ordered by increasing population size.

\begin{tabular}{|c|c|c|c|c|}
\hline Region & $\begin{array}{l}\text { Random } \\
\text { Intercept }\end{array}$ & $\begin{array}{r}\text { Random } \\
\text { Slope }\end{array}$ & M-quantile & Expectile \\
\hline 1 & 3.57 & -6.99 & -14.12 & -0.14 \\
\hline 2 & 12.12 & 15.50 & -22.20 & -12.16 \\
\hline 3 & -4.77 & 0.64 & -29.13 & -14.65 \\
\hline 4 & 30.66 & 10.21 & 6.81 & 15.91 \\
\hline 5 & 9.15 & 5.70 & -12.90 & -0.20 \\
\hline 6 & 50.54 & 22.81 & -21.31 & 0.73 \\
\hline 7 & 1.94 & -2.69 & -10.71 & 2.95 \\
\hline 8 & -10.51 & -7.72 & -16.61 & -15.85 \\
\hline 9 & -7.10 & 4.93 & -22.90 & -21.38 \\
\hline 10 & -4.59 & -2.45 & -14.20 & -10.95 \\
\hline 11 & 15.36 & 14.36 & 2.84 & 12.30 \\
\hline 12 & -7.87 & -8.72 & -31.51 & -24.87 \\
\hline 13 & 7.08 & 8.19 & -12.27 & -5.31 \\
\hline 14 & 1.08 & 2.45 & -10.06 & -3.77 \\
\hline 15 & 2.10 & 0.09 & -14.56 & -7.18 \\
\hline 16 & -6.21 & -7.02 & -30.97 & -25.09 \\
\hline 17 & 4.83 & 5.40 & -14.89 & -8.70 \\
\hline 18 & 25.62 & 32.44 & 10.87 & 18.93 \\
\hline 19 & -2.55 & -2.54 & -21.29 & -14.96 \\
\hline 20 & 12.35 & 12.77 & -4.43 & 12.35 \\
\hline 21 & -10.49 & -8.67 & -26.39 & -20.14 \\
\hline 22 & 4.55 & 1.00 & -15.87 & -3.06 \\
\hline 23 & 6.26 & 6.34 & -8.20 & -1.59 \\
\hline 24 & -7.78 & -5.44 & -31.29 & -25.13 \\
\hline 25 & -6.33 & -3.01 & -29.46 & -24.31 \\
\hline 26 & 2.44 & 3.53 & -15.17 & -9.10 \\
\hline 27 & -2.72 & -4.25 & -23.76 & -18.50 \\
\hline 28 & -0.07 & 0.65 & -16.18 & -9.12 \\
\hline 29 & -1.60 & -2.20 & -19.05 & -13.48 \\
\hline Mean & 4.04 & 2.94 & -16.17 & -7.81 \\
\hline Median & 1.94 & 0.65 & -15.87 & -9.10 \\
\hline
\end{tabular}


Table 4 Relative root mean squared errors of estimates of regional means of TCC in simulation study. Regions ordered by increasing population size.

\begin{tabular}{|c|c|c|c|c|}
\hline Region & $\begin{array}{l}\text { Random } \\
\text { Intercept }\end{array}$ & $\begin{array}{r}\text { Random } \\
\text { Slope }\end{array}$ & M-quantile & Expectile \\
\hline 1 & 19.63 & 31.41 & 24.32 & 24.36 \\
\hline 2 & 22.13 & 41.71 & 31.89 & 25.64 \\
\hline 3 & 21.52 & 28.72 & 30.88 & 23.48 \\
\hline 4 & 36.66 & 20.71 & 15.51 & 20.98 \\
\hline 5 & 17.43 & 15.03 & 14.81 & 10.04 \\
\hline 6 & 98.51 & 57.86 & 37.38 & 40.10 \\
\hline 7 & 17.84 & 26.41 & 13.05 & 16.10 \\
\hline 8 & 14.21 & 12.41 & 17.67 & 16.86 \\
\hline 9 & 14.43 & 16.43 & 23.92 & 24.09 \\
\hline 10 & 10.37 & 10.04 & 15.88 & 13.72 \\
\hline 11 & 21.21 & 21.78 & 7.02 & 13.74 \\
\hline 12 & 15.24 & 14.40 & 31.90 & 25.39 \\
\hline 13 & 15.51 & 16.16 & 13.19 & 7.60 \\
\hline 14 & 11.45 & 19.56 & 13.74 & 11.43 \\
\hline 15 & 18.07 & 17.18 & 15.44 & 9.33 \\
\hline 16 & 11.27 & 11.93 & 31.23 & 25.43 \\
\hline 17 & 16.24 & 16.15 & 15.56 & 10.51 \\
\hline 18 & 34.91 & 43.88 & 14.21 & 21.58 \\
\hline 19 & 7.72 & 8.27 & 21.78 & 15.74 \\
\hline 20 & 20.55 & 21.99 & 12.65 & 18.27 \\
\hline 21 & 29.85 & 28.47 & 26.74 & 21.35 \\
\hline 22 & 12.20 & 9.59 & 17.18 & 12.12 \\
\hline 23 & 17.54 & 17.68 & 9.11 & 4.75 \\
\hline 24 & 17.05 & 18.49 & 31.50 & 25.43 \\
\hline 25 & 11.13 & 9.81 & 29.62 & 24.54 \\
\hline 26 & 11.65 & 11.79 & 15.58 & 9.83 \\
\hline 27 & 8.98 & 11.28 & 24.07 & 19.05 \\
\hline 28 & 7.68 & 7.42 & 16.81 & 10.32 \\
\hline 29 & 7.46 & 7.02 & 19.41 & 14.04 \\
\hline Mean & 19.60 & 19.78 & 20.41 & 17.79 \\
\hline Median & 16.24 & 16.43 & 17.18 & 16.86 \\
\hline
\end{tabular}


Table 5 Relative biases of estimates of regional medians of TCC in simulation study. Regions ordered by increasing population size.

\begin{tabular}{|c|c|c|c|c|}
\hline Region & $\begin{array}{l}\text { Random } \\
\text { Intercept }\end{array}$ & $\begin{array}{r}\text { Random } \\
\text { Slope }\end{array}$ & M-quantile & Expectile \\
\hline 1 & 44.54 & 33.01 & 35.17 & 37.87 \\
\hline 2 & 168.74 & 180.47 & 10.67 & -0.81 \\
\hline 3 & 51.74 & 54.59 & 7.91 & 12.00 \\
\hline 4 & 35.02 & 18.64 & -10.48 & -11.78 \\
\hline 5 & -9.05 & 9.27 & -11.01 & -20.95 \\
\hline 6 & -4.16 & 36.28 & -0.27 & -0.67 \\
\hline 7 & 5.06 & 12.61 & 8.38 & 5.83 \\
\hline 8 & -3.73 & -4.30 & -5.88 & -3.96 \\
\hline 9 & 10.89 & 11.15 & -12.21 & -14.21 \\
\hline 10 & -14.56 & -14.19 & -22.31 & -17.61 \\
\hline 11 & 71.73 & 62.21 & 39.27 & 40.21 \\
\hline 12 & 35.05 & 22.09 & 6.48 & 6.42 \\
\hline 13 & 15.18 & 18.79 & -12.48 & -13.63 \\
\hline 14 & 7.99 & 7.23 & 1.11 & 1.59 \\
\hline 15 & 4.32 & 4.51 & -3.92 & -4.77 \\
\hline 16 & 58.15 & 50.72 & 6.36 & 5.01 \\
\hline 17 & 28.28 & 29.52 & -0.51 & -0.62 \\
\hline 18 & 17.82 & 34.61 & -15.52 & -16.91 \\
\hline 19 & 30.91 & 29.72 & 6.69 & 8.10 \\
\hline 20 & 68.38 & 77.64 & 59.60 & 60.68 \\
\hline 21 & 55.26 & 67.53 & 10.40 & 11.59 \\
\hline 22 & 94.74 & 86.35 & 53.58 & 52.79 \\
\hline 23 & 7.06 & 15.12 & -9.14 & -10.16 \\
\hline 24 & 28.40 & 23.99 & -3.50 & -6.83 \\
\hline 25 & 29.56 & 25.51 & 7.59 & 2.51 \\
\hline 26 & 30.50 & 31.67 & 13.29 & 10.68 \\
\hline 27 & 15.47 & 9.65 & -11.11 & -11.77 \\
\hline 28 & 8.01 & 7.08 & -1.64 & -2.74 \\
\hline 29 & 6.56 & 6.37 & -10.53 & -11.15 \\
\hline Mean & 30.96 & 32.68 & 4.69 & 3.68 \\
\hline Median & 28.30 & 24.00 & -0.27 & -0.67 \\
\hline
\end{tabular}


Table 6 Relative median biases of estimates of regional medians of TCC in simulation study. Regions ordered by increasing population size.

\begin{tabular}{|c|c|c|c|c|}
\hline Region & $\begin{array}{l}\text { Random } \\
\text { Intercept }\end{array}$ & $\begin{array}{r}\text { Random } \\
\text { Slope }\end{array}$ & M-quantile & Expectile \\
\hline 1 & 44.78 & 29.46 & 10.68 & 11.92 \\
\hline 2 & 166.14 & 183.77 & -7.63 & -30.63 \\
\hline 3 & 50.76 & 50.38 & 0.13 & 8.18 \\
\hline 4 & 33.63 & 16.03 & -17.37 & -17.53 \\
\hline 5 & -5.74 & 7.96 & -13.78 & -24.66 \\
\hline 6 & 3.79 & 28.73 & -0.51 & 0.26 \\
\hline 7 & 3.53 & 8.90 & 8.05 & 3.55 \\
\hline 8 & -3.31 & -4.26 & -6.78 & -5.53 \\
\hline 9 & 9.38 & 9.54 & -8.46 & -12.04 \\
\hline 10 & -15.41 & -14.94 & -21.61 & -18.77 \\
\hline 11 & 72.19 & 56.07 & 35.84 & 34.91 \\
\hline 12 & 32.86 & 21.65 & 4.79 & 3.70 \\
\hline 13 & 9.99 & 14.30 & -12.38 & -12.93 \\
\hline 14 & 9.65 & 7.56 & 0.66 & -1.32 \\
\hline 15 & -4.62 & -2.04 & -3.80 & -5.06 \\
\hline 16 & 56.15 & 48.25 & 3.89 & 4.03 \\
\hline 17 & 21.93 & 24.33 & 0.02 & 0.02 \\
\hline 18 & 16.83 & 33.17 & -13.85 & -15.64 \\
\hline 19 & 30.42 & 29.17 & 5.10 & 6.91 \\
\hline 20 & 65.96 & 76.38 & 54.86 & 54.78 \\
\hline 21 & 38.13 & 52.32 & 8.94 & 9.81 \\
\hline 22 & 104.76 & 84.31 & 54.32 & 53.32 \\
\hline 23 & 0.49 & 8.25 & -9.70 & -11.52 \\
\hline 24 & 24.47 & 17.88 & -4.08 & -7.01 \\
\hline 25 & 29.24 & 25.80 & 7.46 & 2.42 \\
\hline 26 & 27.33 & 28.89 & 13.82 & 11.66 \\
\hline 27 & 13.34 & 8.48 & -12.51 & -13.99 \\
\hline 28 & 7.02 & 6.47 & -1.42 & -2.46 \\
\hline 29 & 5.55 & 5.30 & -11.11 & -11.61 \\
\hline Mean & 29.28 & 29.73 & 2.19 & 0.51 \\
\hline Median & 21.90 & 21.60 & -0.51 & -1.32 \\
\hline
\end{tabular}


Table 7 Relative root mean squared errors of estimates of regional medians of TCC in simulation study. Regions ordered by increasing population size.

\begin{tabular}{|c|c|c|c|c|}
\hline Region & $\begin{array}{l}\text { Random } \\
\text { Intercept }\end{array}$ & $\begin{array}{r}\text { Random } \\
\text { Slope }\end{array}$ & M-quantile & Expectile \\
\hline 1 & 52.16 & 54.11 & 75.28 & 75.19 \\
\hline 2 & 182.04 & 209.03 & 68.38 & 76.09 \\
\hline 3 & 56.07 & 62.07 & 19.53 & 21.49 \\
\hline 4 & 43.16 & 28.77 & 24.66 & 27.89 \\
\hline 5 & 27.30 & 19.25 & 19.26 & 27.05 \\
\hline 6 & 58.83 & 59.45 & 5.24 & 7.10 \\
\hline 7 & 24.30 & 24.03 & 11.28 & 12.78 \\
\hline 8 & 10.88 & 10.93 & 12.65 & 12.27 \\
\hline 9 & 17.87 & 17.10 & 20.87 & 22.86 \\
\hline 10 & 17.68 & 17.70 & 25.97 & 21.42 \\
\hline 11 & 76.12 & 67.71 & 44.13 & 46.27 \\
\hline 12 & 40.16 & 27.27 & 17.48 & 16.54 \\
\hline 13 & 25.70 & 27.09 & 15.17 & 16.25 \\
\hline 14 & 13.90 & 13.67 & 15.20 & 14.60 \\
\hline 15 & 34.73 & 32.65 & 11.57 & 12.00 \\
\hline 16 & 61.45 & 53.76 & 15.61 & 13.97 \\
\hline 17 & 37.90 & 38.22 & 6.70 & 7.85 \\
\hline 18 & 37.97 & 53.96 & 23.36 & 24.26 \\
\hline 19 & 33.09 & 31.76 & 11.42 & 13.16 \\
\hline 20 & 80.66 & 85.74 & 67.38 & 68.66 \\
\hline 21 & 92.25 & 96.63 & 17.12 & 18.34 \\
\hline 22 & 104.68 & 90.06 & 56.77 & 59.34 \\
\hline 23 & 31.01 & 31.70 & 12.70 & 14.03 \\
\hline 24 & 36.82 & 35.90 & 8.46 & 11.56 \\
\hline 25 & 33.30 & 28.18 & 11.60 & 10.59 \\
\hline 26 & 35.50 & 36.00 & 14.95 & 13.78 \\
\hline 27 & 21.48 & 19.42 & 12.46 & 13.44 \\
\hline 28 & 13.78 & 12.24 & 5.60 & 6.09 \\
\hline 29 & 11.96 & 10.29 & 11.72 & 12.18 \\
\hline Mean & 45.27 & 44.65 & 22.85 & 24.04 \\
\hline Median & 35.50 & 31.76 & 15.20 & 14.60 \\
\hline
\end{tabular}


Table 8 Coverage rates of "2 sigma" confidence intervals for regional population means of TCC. Intervals are defined by the M-quantile and Expectile estimates plus or minus twice their corresponding standard errors using either (8a) ("Population Residuals") or (8b) ("Area Residuals") in (10). Regions ordered by increasing population size.

\begin{tabular}{|c|c|c|c|c|}
\hline \multirow[t]{2}{*}{ Region } & \multicolumn{2}{|c|}{ Area Residuals } & \multicolumn{2}{|c|}{ Population Residuals } \\
\hline & M-quantile & Expectile & M-quantile & Expectile \\
\hline 1 & 0.56 & 0.84 & 0.55 & 0.88 \\
\hline 2 & 1.00 & 0.97 & 1.00 & 0.98 \\
\hline 3 & 0.46 & 0.79 & 0.40 & 0.78 \\
\hline 4 & 0.98 & 1.00 & 0.99 & 1.00 \\
\hline 5 & 0.93 & 1.00 & 0.96 & 1.00 \\
\hline 6 & 0.94 & 0.99 & 0.95 & 1.00 \\
\hline 7 & 0.82 & 0.99 & 0.75 & 0.99 \\
\hline 8 & 0.99 & 1.00 & 0.99 & 1.00 \\
\hline 9 & 0.47 & 1.00 & 0.58 & 1.00 \\
\hline 10 & 0.99 & 1.00 & 0.99 & 1.00 \\
\hline 11 & 1.00 & 1.00 & 1.00 & 1.00 \\
\hline 12 & 0.21 & 0.65 & 0.17 & 0.67 \\
\hline 13 & 1.00 & 1.00 & 1.00 & 1.00 \\
\hline 14 & 0.96 & 1.00 & 0.94 & 1.00 \\
\hline 15 & 0.60 & 0.94 & 0.56 & 0.96 \\
\hline 16 & 0.03 & 0.56 & 0.03 & 0.78 \\
\hline 17 & 0.52 & 1.00 & 0.63 & 1.00 \\
\hline 18 & 1.00 & 1.00 & 1.00 & 1.00 \\
\hline 19 & 1.00 & 1.00 & 1.00 & 1.00 \\
\hline 20 & 1.00 & 1.00 & 1.00 & 1.00 \\
\hline 21 & 0.96 & 1.00 & 0.99 & 1.00 \\
\hline 22 & 1.00 & 1.00 & 1.00 & 1.00 \\
\hline 23 & 1.00 & 1.00 & 1.00 & 1.00 \\
\hline 24 & 0.06 & 0.59 & 0.06 & 0.76 \\
\hline 25 & 0.26 & 0.98 & 0.19 & 0.99 \\
\hline 26 & 0.99 & 1.00 & 1.00 & 1.00 \\
\hline 27 & 0.77 & 1.00 & 0.68 & 0.99 \\
\hline 28 & 1.00 & 1.00 & 1.00 & 1.00 \\
\hline 29 & 1.00 & 1.00 & 1.00 & 1.00 \\
\hline Mean & 0.77 & 0.94 & 0.77 & 0.96 \\
\hline Median & 0.94 & 1.00 & 0.99 & 1.00 \\
\hline
\end{tabular}

